\title{
Impact of Inter-Pregnancy Interval on Long-Term Endo-Metabolic Health of the Offspring
}

\author{
Seidman Liron'), Wainstock Tamar'), Sheiner Eyal3), Pariente Gali4), \\ Sergienko Ruslan5), Walfisch Asnat6) \\ 1)The Goldman Medical School at the Faculty of Health Sciences, \\ Ben-Gurion University of the Negev, Beer-Sheva, Israel. \\ 2)Ben-Gurion University of the Negev, Department of Epidemiology \\ and Health Services Evaluation, Beer-Sheva, Israel. \\ 3)Department of Obstetrics and Gynecology, Soroka University Medical Center, \\ Faculty of Health Sciences, Ben-Gurion University of the Negev, Beer-Sheva, Israel. \\ 4)Department of Obstetrics and Gynecology, Soroka University Medical Center, \\ Faculty of Health Sciences, Ben-Gurion University of the Negev, Beer-Sheva, Israel. \\ 5)Ben-Gurion University of the Negev, Department of Epidemiology \\ and Health Services Evaluation, Beer-Sheva, Israel. \\ 6)Department of Obstetrics and Gynecology, Soroka University Medical Center, \\ Faculty of Health Sciences, Ben-Gurion University of the Negev, Beer-Sheva, Israel
}

\section{ABSTRACT}

Background: Inter-pregnancy interval (IPI), defined as the time interval between a live birth and estimated conception time of a subsequent pregnancy, has an established effect on perinatal outcome. The long-term impact of IPI on offspring is unknown. This study aimed to examine the effect of short and long IPIs on longterm endo-metabolic health of offspring.

Subjects and Method: This populationbased cohort study included singleton live births in parturient with at least one previous birth. Singleton deliveries between the years 1991-2014 in a regional tertiary medical center were included. Offspring were followed until 18 years of age for endo-metabolic related hospitalizations. Survival curves were used to compare cumulative incidence of endo-metabolic morbidity, and Cox proportional hazards models to control for confounders.

Results: During the study period 144,397 deliveries met the inclusion criteria. Offspring following long IPIs exhibited higher rates of endometabolic related hospitalizations. Survival curCite this as:

Liron S, Tamar W, Eyal S, Gali P, Ruslan S, Asnat W (2020). Impact of Inter-Pregnancy Interval on LongTerm Endo-Metabolic Health of the Offspring. J Matern Child Health. 05(03): 321-330. https://doi.org/10.26911/thejmch.2020.05.03.11. al and Child Health is licensed under a Creative Commons

Attribution-NonCommercial-ShareAlike 4.0 International License.

\section{BACKGROUND}

Interpregnancy interval (IPI) is the period between the birth of a live newborn and the ve demonstrated a significantly higher cumulative incidence of endo-metabolic morbidity in the long IPI group $(\mathrm{p}<0.001)$. The Cox model demonstrated long IPI to significantly increase the risk for endo-metabolic related hospitalizations during childhood $(\mathrm{aHR}=1.34,95 \% \mathrm{CI}=$ 1.06 to $1.70 ; \mathrm{p}=0.015$ ).

Conclusion: Long IPI appears to have an independent impact on long-term endo-metabolic health of the offspring.

Keywords: pregnancy interval, offspring, endocrine health, metabolic morbidity, obesity

\section{Correspondence:}

Liron Seidman. The Goldman Medical School at the Faculty of Health Sciences, Ben-Gurion University of the Negev. Ben-Gurion University Medical School, Soroka University Medical Center, 151 IzakRager Ave, Be'er-Sheva 84101, Israel. Email: lironseidman@gmail.com. Mobile: +972-524215808

conception of the next sibling (Class et al., 2017). Various definitions have been used to define IPI. One commonly used defini- 
Seidman et al./ Inter-pregnancy interval on long-term endo-metabolic health

tion for "short IPI" refers to an interval of less than 18 months. Other researchers, however, have referred to a "short IPI" within a shorter range of less than three to six months (Li et al., 2018). There seems to be wide agreement regarding the definition of "long IPI," which is frequently defined as an IPI longer than 59 or 60 months (Conde-Agudelo et al., 2006).

The possible impact of IPI on perinatal outcomes has been investigated since the early 1920s (Li et al., 2018). A deviation from an average IPI length was shown to be associated with adverse outcomes in offspring. As IPI is a potentially modifiable risk factor, it attracted significant attention from the medical community (Li et al., 2018).

Both short and long IPIs have been reported to be associated with a significantly increased risk of adverse perinatal outcomes, such as preterm delivery (PTD), low birth weight (LBW<2500 g), small for gestational age (SGA) and perinatal death (Conde-Agudelo et al., 2006). For instance, Ratzon et al. (2011) showed that short IPI increases the risk of adverse pregnancy outcomes such as recurrent preterm delivery, and Class et al. (2017) found that long IPI increases the risk of PTD by $51 \%$, the risk of LBW by $61 \%$, and the incidence of SGA by $54 \%$.

Studies suggest a difference in the direct influence of short or long IPIs. Short IPI associated adverse perinatal outcomes are largely attributed to familial confounding. The association of long IPI with infant morbidities has more often been suggested to be independent of measured and unmeasured confounding factors (Class et al., 2017).

It seems that little is currently known about the influence of IPI on long-term childhood growth, development and health. This stands in contrast with the current growing interest and numerous publications on perinatal exposures and their impact on long term health of offspring (Barker et al., 2002; De Boo and Harding, 2006).

Some investigators explored the impact of several pregnancy and maternal characteristics on the endo-metabolic health of their offspring (Paz Levy et al., 2017; Spiegel et al., 2019). Maternal characteristics include adverse early pregnancy lipid profile and increased pre-pregnancy body mass index (BMI) (Oostvogels et al., 2014; van Lieshout et al., 2017), as well as maternal diabetes or hypothyroidism (Abokaf et al., 2018; Eshkoli et al., 2019). Some authors suggested these exposures to elevate the risk of later offspring metabolic health (van Lieshout et al., 2017). Childhood obesity has become a significant world-wide epidemic with important social and health burdens (Tran et al., 2019).In light of the disturbing increase over the last decade in the incidence of endo-metabolic disorders in children, the authors decided to examine this association. Our aim was to examine the effect of short ( $<6$ months) and long ( $>60$ months) IPIs on long-term endo-metabolic health of the offspring in a large population-based cohort study.

\section{SUBJECTS AND METHOD}

\section{Study Design}

This population-based retrospective cohort study was conducted at the Soroka University Medical Center (SUMC), the sole tertiary medical center in the Negev (southern region of Israel). Thus, SUMC serves the entire population of the region, 750,000 inhabitants in 2018 (Bureau and Statistics, 2019). Since SUMC is the major medical center in the south of Israel, most of the children born at SUMC are expected to be hospitalized there, if the need arises. 
Seidman et al./ Inter-pregnancy interval on long-term endo-metabolic health

\section{Population and Sample}

All singleton pregnancies of women who delivered at the SUMC and their infants (up to the age of 18 years) between January 1991 and January 2014. Thus, the study is based on non-selective population data.

The researchers excluded from the study women who delivered only once, multiple pregnancies, unknown gestational age, fetuses with major congenital malformations or chromosomal abnormalities, and all perinatal mortality cases (intrauterine fetal death, intra-partum death and post-partum death).

\section{Operational Definition of Variables}

Short IPI was defined as less than 6 months, intermediate IPI (between 6 to 60 months), and long IPI was defined as an IPI of (more than 60 months).

The variables obtained for the analysis included the following maternal factors: maternal age, parity, hypertensive disorders in pregnancy, diabetes in pregnancy, and maternal obesity. Perinatal factors obtained included: gestational age and preterm delivery, and birthweight.

The outcome variables included the following major pediatric metabolic diseases: diabetes mellitus, obesity, sex hormone disease, hypoglycemia and hyperlipidemia. All diagnoses are classified by the international classification of disease (ICD-9, appendix A).

\section{Study Instruments}

In this population-based retrospective cohort study, data were collected from two computerized databases that were crosslinked and merged. The first, was the perinatal database of the Obstetrics and Gynecology department in SUMC, including information documented by obstetricians following each delivery. Experienced medical secretaries routinely review the information before entering it into the database to ensure its maximal completeness and accuracy. Coding was performed after assessing medical prenatal care records as well as routine hospital documents.

The second, was the SUMC general hospitalization records for children up to the age of 18 years, which includes demographic data and medical diagnoses obtained upon admission and during hospitalization.

\section{Data Analysis}

Univariable analysis was performed to compare background characteristics, and endocrine and metabolic morbidity, between the three study groups.

The univariable analysis included Pearson Chi-square tests for categorical variables and ANOVA for continuous variables with normal distribution. The comparison was performed across all levels of exposure: short, intermediate and long IPI). Cumulative incidence rates of endocrine and metabolic related hospitalizations of the offspring were compared using KaplanMeier survival curve, using the log-rank test to determine significant differences. Two dummy variables were defined as short and long IPI groups. Those variables were created to compare the short and long IPI groups to the intermediate reference group.

Cox proportional survival hazards model was conducted to compare endometabolic associated hospitalizations risk among offspring born following a short or a long IPI. The model was adjusted for potential confounders based on the univariable analysis, and on clinical importance of the variables. The confounders included in the regression model were maternal factors (age, diabetes mellitus, obesity and hypertensive disorders), as well as obstetrical factors (birthweight and gestational age upon delivery). All analyses were performed using SPSS (version 23) or STATA (version 12) software. All analyses were 2-sided; and 
a $\mathrm{p}$ value of less than 0.05 was considered significant.

\section{Research Ethics}

The institutional review board, in accordance with the Helsinki declaration, approved the study.

\section{RESULTS}

\section{Sample Characteristics}

The inclusion criteria were met by 161,791 deliveries during the study period. Of those 22,851 (14.1\%) occurred in women with short IPIs, $127,144(78.6 \%)$ in women with intermediate IPIs, and 11,796 (7.3\%) in women with long IPIs. Table 1 summarizes maternal characteristic for the different IPI categories. Mean maternal age upon (IPI)

\begin{tabular}{lcccccc}
\hline \multirow{2}{*}{ Characteristics } & \multicolumn{2}{c}{$\begin{array}{c}\text { Long IPI } \\
\text { (>60 months) }\end{array}$} & \multicolumn{2}{c}{$\begin{array}{c}\text { Intermediate IPI } \\
\text { (6-6o months) }\end{array}$} & \multicolumn{2}{c}{$\begin{array}{c}\text { Short IPI } \\
\text { (<6 months) }\end{array}$} \\
\cline { 2 - 7 } & Mean & SD & Mean & SD & Mean & SD \\
\hline Maternal age (year) & 34.50 & 4.28 & 29.2 & 5.33 & 26.14 & 5.07 \\
\hline
\end{tabular}

Table 2. Maternal characteristics (categorical data) by interpregnancy interval (IPI)

\begin{tabular}{lcccccc}
\hline \multirow{2}{*}{ Characteristics } & \multicolumn{2}{c}{$\begin{array}{c}\text { Long IPI } \\
\text { (> 6o months) }\end{array}$} & \multicolumn{2}{c}{$\begin{array}{c}\text { Intermediate IPI } \\
\text { (6-6o months) }\end{array}$} & \multicolumn{2}{c}{$\begin{array}{c}\text { Short IPI } \\
\text { (<6 months) }\end{array}$} \\
\cline { 2 - 7 } & $\mathbf{n}$ & $\mathbf{\%}$ & $\mathbf{n}$ & $\mathbf{\%}$ & $\mathbf{n}$ & $\mathbf{\%}$ \\
\hline Parity & & & & & & \\
2 to 4 & 9,241 & 78.3 & 80,938 & 63.7 & 15,829 & 69.3 \\
$5^{+}$ & 2,555 & 21.7 & 46,208 & 36.3 & 7,022 & 30.7 \\
Hypertensive disorder & 810 & 6.9 & 5,172 & 4.1 & 615 & 2.7 \\
Diabetes in Pregnancy** & 1,202 & 10.2 & 6,251 & 4.9 & 673 & 2.9 \\
Obesity (BMI $\left.\geq 30 \mathrm{Kg} / \mathrm{m}^{2}\right)$ & 167 & 1.4 & 1340 & 1.1 & 189 & 0.8 \\
\hline
\end{tabular}

${ }^{*}$ Including pre-gestational, gestational hypertension, and preeclampsia.

** Including pre-gestational and gestational diabetes mellitus.

\section{The result of bivariate analysis}

Long-term endocrine and metabolic hospitalization rates of offspring according to maternal IPI duration, are presented in Table 3. During the 18-year follow-up period, 690 children were hospitalized with endocrine and metabolic morbidity. Children born following long IPIs exhibited higher rates of endo-metabolic related hospitalizations, as compared with the intermediate and short IPI groups (0.8\% vs. $0.5 \%$ vs. $0.4 \%$ respectively). Specifically, diabetes mellitus and obesity were more common in offspring born following long IPI. 
Seidman et al./ Inter-pregnancy interval on long-term endo-metabolic health

Table 3. Pregnancy outcomes (continous data) by interpregnancy interval (IPI)

\begin{tabular}{|c|c|c|c|c|c|c|c|}
\hline \multirow[t]{2}{*}{ Outcomes } & \multicolumn{2}{|c|}{$\begin{array}{c}\text { Short IPI } \\
\text { (<6 months) }\end{array}$} & \multicolumn{2}{|c|}{$\begin{array}{c}\text { Intermediate IPI } \\
\text { (6-60 months) }\end{array}$} & \multicolumn{2}{|c|}{$\begin{array}{c}\text { Long IPI } \\
\text { (>60 months) }\end{array}$} & \multirow[t]{2}{*}{$\mathbf{p}$} \\
\hline & Mean & SD & Mean & SD & Mean & SD & \\
\hline Birth weight (gram) & $3,171.2$ & 487.4 & $3,258.1$ & 497.5 & $3,235.6$ & 516.2 & $<0.001$ \\
\hline Gestational age (weeks) & 39.06 & 1.97 & 39.18 & 1.92 & 38.85 & 2.02 & $<0.001$ \\
\hline
\end{tabular}

Table 4. Pregnancy outcomes (categorical data) by interpregnancy interval (IPI)

\begin{tabular}{|c|c|c|c|c|c|c|c|}
\hline \multirow[t]{2}{*}{ Characteristics } & \multicolumn{2}{|c|}{$\begin{array}{c}\text { Short IPI } \\
\text { ( }<6 \text { months })\end{array}$} & \multicolumn{2}{|c|}{$\begin{array}{c}\text { Intermediat } \\
\text { e IPI (6-60 } \\
\text { months) }\end{array}$} & \multicolumn{2}{|c|}{$\begin{array}{c}\text { Long IPI } \\
\text { (>60 months) }\end{array}$} & \multirow[t]{2}{*}{$\mathbf{p}$} \\
\hline & $\mathbf{n}$ & $\%$ & $\mathbf{n}$ & $\%$ & $\mathbf{n}$ & \% & \\
\hline \multicolumn{8}{|l|}{ Gender } \\
\hline Male & 1,575 & 50.7 & 64,656 & 50.9 & 5,925 & 50.1 & 0.315 \\
\hline Female & 1,276 & $49 \cdot 3$ & 62,490 & 49.1 & 5,881 & 49.9 & \\
\hline Preterm delivery & 1,799 & $7 \cdot 9$ & 7,498 & $5 \cdot 9$ & 909 & $7 \cdot 7$ & $<0.001$ \\
\hline Low birth weight $(<2500$ g) & 1,569 & 6.9 & 6,637 & 5.2 & 782 & 6.6 & $<0.001$ \\
\hline Apgar score $<7$ at $1 \mathrm{~min}$ & 1,272 & 5.6 & 6,374 & 5 & 550 & $4 \cdot 7$ & $<0.001$ \\
\hline Apgar score $<7$ at $5 \mathrm{~min}$ & 786 & 3.4 & 3,088 & 2.4 & 162 & 1.4 & $<0.001$ \\
\hline
\end{tabular}

Table 5. Selected long-term endocrine and metabolic morbidity of offspring born stratified by different interpregnancy interval (IPI) lengths.

\begin{tabular}{|c|c|c|c|c|c|c|c|}
\hline \multirow{2}{*}{$\begin{array}{l}\text { Offspring Long-Term } \\
\text { Morbidity }\end{array}$} & \multicolumn{2}{|c|}{$\begin{array}{c}\text { Short IPI } \\
\text { (<6 months) }\end{array}$} & \multicolumn{2}{|c|}{$\begin{array}{l}\text { Intermediate IPI } \\
\text { (6-60 months) }\end{array}$} & \multicolumn{2}{|c|}{$\begin{array}{c}\text { Long IPI } \\
\text { (>60 months) }\end{array}$} & \multirow[t]{2}{*}{$\mathbf{p}$} \\
\hline & $\mathbf{n}$ & $\%$ & $\mathbf{n}$ & $\%$ & $\mathbf{n}$ & $\%$ & \\
\hline Thyroid disease & 7 & 0.03 & 59 & 0.1 & 6 & 0.1 & 0.694 \\
\hline Diabetes mellitus & 10 & 0.1 & 110 & 0.1 & 16 & 0.1 & 0.048 \\
\hline Hypoglycemia & 23 & 0.1 & 138 & 0.1 & 21 & 0.3 & 0.195 \\
\hline Hyperlipidemia & 0 & 0 & 1 & 0.0008 & $\mathrm{O}$ & 0 & 0.875 \\
\hline Obesity & 30 & 0.2 & 180 & 0.2 & 38 & 0.3 & $<0.001$ \\
\hline Parathyroid disease & 3 & 0.0001 & 21 & 0.0002 & 3 & 0.0003 & 0.805 \\
\hline Adrenal disease & 4 & 0.0002 & 20 & 0.02 & 4 & 0.0003 & 0.943 \\
\hline Sex hormone disease & o & o & 11 & 0.0001 & 4 & 0.0003 & 0.013 \\
\hline $\begin{array}{l}\text { Total endo-metabolic } \\
\text { related hospitalization }\end{array}$ & 75 & 0.4 & 528 & 0.5 & 87 & 0.8 & $<0.001$ \\
\hline
\end{tabular}

\section{The result of multivariate analysis}

The Kaplan-Meier survival curve (Figure 1) demonstrated a significantly higher cumulative incidence of long-term endocrine and metabolic morbidity in the long IPI group (log rank $\mathrm{p}<0.001$ ). Table 4 presents the Cox proportional hazards model for the association between IPI and offspring long term endocrine and metabolic morbidity.
The model controlled for maternal age, diabetes mellitus, hypertensive disorders, and obesity, as well as preterm delivery and birthweight. The model confirmed that infants born following long IPIs were at a significant and independent risk for longterm endocrine and metabolic related hospitalizations during childhood (adjusted $\mathrm{HR}=1.34,95 \% \mathrm{CI}=1.06$ to $1.70 ; \mathrm{p}=0.015$ ). 


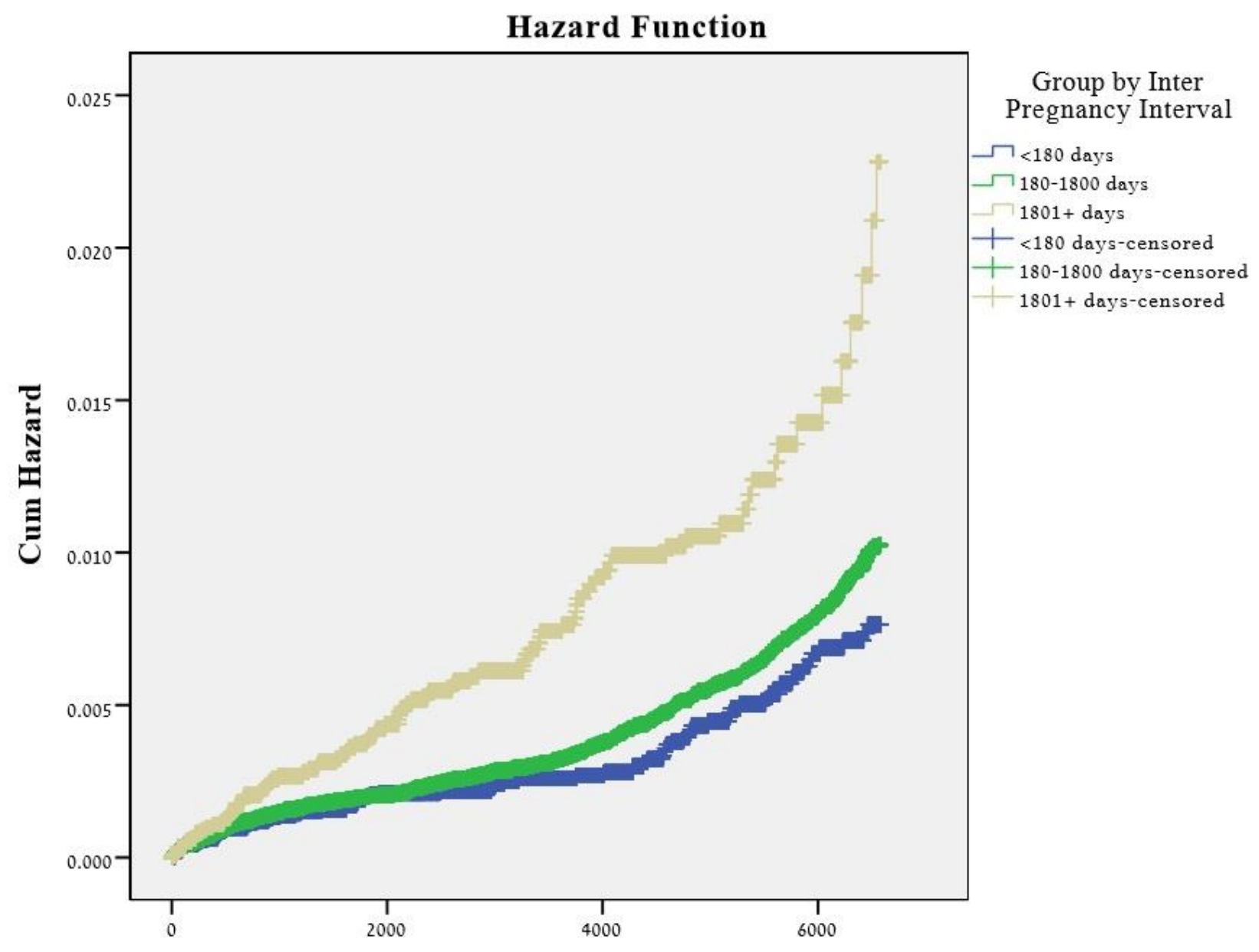

Followup time in days

Figure 1. Kaplan-Meier survival curve for the cumulative incidence of endocrine and metabolic hospitalizations over time, from birth up to 18 years of age, according to inter pregnancy interval

(log-rank p<0.001).

Table 6. Multivariable analysis for the association between interpregnancy interval (IPI) and offspring long term endocrine and metabolic morbidity.

\begin{tabular}{|c|c|c|c|c|}
\hline \multirow[b]{2}{*}{ Independent Variables } & \multirow{2}{*}{$\begin{array}{c}\text { adjusted Hazard } \\
\text { Ratio }\end{array}$} & \multicolumn{2}{|c|}{$95 \% \mathrm{CI}$} & \multirow[b]{2}{*}{$\mathbf{p}$} \\
\hline & & $\begin{array}{l}\text { Lower } \\
\text { limit }\end{array}$ & $\begin{array}{c}\text { Upper } \\
\text { limit }\end{array}$ & \\
\hline \multicolumn{5}{|l|}{ Intermediate IPI } \\
\hline Long IPI (>60 months) & 1.34 & 1.06 & 1.70 & 0.015 \\
\hline Short IPI ( $<6$ months) & 1.01 & 0.79 & 1.31 & 0.88 \\
\hline Maternal age (years) & 0.96 & 0.94 & 0.97 & $<0.001$ \\
\hline Preterm delivery ( $<37$ weeks) & 1.28 & 0.96 & 1.72 & 0.1 \\
\hline Birthweight (grams) & 0.99 & 0.99 & 1.00 & 0.12 \\
\hline Hypertensive disorders of pregnancy & 1.21 & 0.89 & 1.66 & 0.23 \\
\hline $\begin{array}{l}\text { Diabetes mellitus (gestational and pre- } \\
\text { gestational) }\end{array}$ & 1.67 & 1.29 & 2.16 & $<0.001$ \\
\hline Obesity $\left(\mathrm{BMI} \geq 30 \mathrm{Kg} / \mathrm{m}^{2}\right)$ & 2.62 & 1.72 & 3.98 & $<0.001$ \\
\hline
\end{tabular}




\section{DISCUSSION}

The researchers found that long IPI is strongly associated with an increased incidence of long-term endocrine and metabolic morbidity of the offspring. Specifically, a significantly increased risk for diabetes mellitus and obesity in offspring born following long IPIs was demonstrated, as compared with the intermediate and short IPI groups. This original observation raises the question of whether long IPI represents an independent risk factor or whether this association can be explained by maternal and obstetrical confounding factors. Due to the large size of our cohort, the researchers were able to design a multiple logistic regression analysis controlling for many potential confounding factors. Factors controlled for in our model included maternal factors like age, diabetes mellitus, obesity and hypertensive disorders, and obstetrical factors such as birthweight and preterm delivery.

Current published literature focuses mainly on the significant effect of a short IPI and adverse perinatal outcome. For instance, short IPI had been associated with pre-term delivery, neonatal and fetal death, LBW, and SGA (Conde-Agudelo et al., 2006; Mahande and Obure, 2016; Cecatti et al., 2008; Mignini et al., 2016). Long IPI, however, has been less investigated, yet some important associations have been suggested, including the risk for preterm delivery, LBW, SGA, fetal death, and increased odds of pre-eclampsia (Cecatti et al., 2008; Class et al., 2017; Mahande and Obure, 2016; Cecatti et al., 2008; Mignini et al., 2016; Kaul et al., 2019). As for the association between IPI and long-term health of the offspring, data is scarce. Li et al. (2018) suggested that long IPI is a risk factor for higher diastolic BP in offspring, which can potentially be explained by the confounding effect of advanced maternal age (Li et al., 2018).

The prevailing view today is that endocrine and metabolic disease is not only determined by one's genes, but also by the prenatal (i.e. intra-uterine) environment. This is in line with the currently widely accepted Barker hypothesis. Barker claimed that the fetus can be expected to make physiological adaptations in response to changes in its environment, in order to prepare itself for postnatal life (De Boo and Harding, 2006). For example, Barker's 'thrifty phenotype' hypothesis suggested that adult insulin resistance and type 2 diabetes could result from the persistence of a fetal glucose conserving adaptation in response to intrauterine hypoglycemia (De Boo and Harding, 2006). This is consistent with a recent study suggesting an association between in utero exposure to gestational diabetes (GDM) and long-term infant endocrine and metabolic morbidity, e.g. higher blood pressure and a predisposition for obesity, impaired glucose tolerance, diabetes, and metabolic syndrome (Abokaf et al., 2018).

Long IPI is strongly linked with factors that could influence the prenatal environment. For instance, maternal age and weight, as well as nutritional status. The association between IPI and endometabolic morbidity has not been previously studied. Therefore, the possibility that long IPI is independently linked with offspring endocrine and metabolic morbidity during childhood is of significant public health interest, as birth spacing is recognized as a modifiable risk factor (Regan et al., 2019).

The major strength of the present study is the large sample upon which it is based. This allowed us to control for several important confounding factors. Although the retrospective nature of this study is an 
obvious limitation, the data was collected prospectively and recorded in real-time. Moreover, this study is based on nonselective population data. The authors used pre-tested, standardized data collection forms, completed by trained data collectors, and a methodology based on a previous WHO global survey (Shah, 2008). Furthermore, since our medical center is the only one in our region, most children born in our hospital are likely to return to our center for medical treatment if warranted. The authors therefore believe that as a regional hospital our study population included a relatively unbiased participation of mothers from all socioeconomic backgrounds. It is also apparent that a randomized controlled study regarding the effects of IPI will not be possible, as mothers are not likely to agree to base their family planning decisions solely on a random study allocation.

This study has several limitations. First, it is based on a large hospital-based database that does not include outpatient visits at non-hospital affiliated clinics. Therefore, cases of endocrine and metabolic disease managed solely in an outpatient setting were not accounted for. Furthermore, although our population is relatively non-mobile, it is clear that some patients may have moved to other regions and therefore missed, although these two limitations are true for all three study groups.

Since this is a population-based analysis, our study can provide evidence only of an association rather than causation. Several potentially important factors were not available, such as childhood exposures, breast feeding, maternal weight or body mass index and socio-economic status. These factors have previously been shown to affect or protect the risk of endocrine and metabolic diseases (Boney et al., 2005; Kaul et al., 2019). For in-stance, lack of breastfeeding in the first 5 months of life was associated with higher likelihood of being overweight in childhood (Boney et al., 2005).

In conclusion, our observation, that long IPI is significantly associated with an increase in long-term endocrine and metabolic morbidity of the offspring, adds an important perspective to be considered during family planning. It seems that women can now be advised that prolonging the interval between births may increase endocrine and metabolic risk in their child. Further study is needed to assess whether this association can be atenuated by interventions such as healthy lifestyle, weight maintenance, or physical activity. It remains to be seen whether counseling to optimize birth spacing can significantly reduce endocrine and metabolic mortality, and improve other immediate and long term pregnancy outcomes (Class et al., 2017; Li et al., 2018; Conde-Agudelo et al., 2006).

Better understanding of this association may therefore lead to practical public health recommendations and interventions, within the realm of family planning and disease prevention, in both the developed and developing world.

\section{AUTHOR CONTRIBUTION}

Seidman L. has participated in all phases of this study including planning, literature search, data interpretation, and has written the first draft of the manuscript. Wainstock $\mathrm{T}$. is a specialist in performing all aspects of the statistical analysis needed for this study. She has participated in all phases of this study and has performed most of the analyses presented in this paper. Sheiner E. has initiated the study and supervised actively throughout its conduct. Specifically, he was involved in the data inter- 
Seidman et al./ Inter-pregnancy interval on long-term endo-metabolic health

pretation, statistical analysis, and has revised the manuscript. Pariente G. has participated in all phases of this study including literature search and research planning, data collection and review, and took part in the data interpretation. Sergienko R. managed data collection, built the database and planned the statistical analysis. Walfisch A. has initiated and participated in all phases of this study including study planning, literature search, data collection and review, interpretation of the results, and has supervised and extensively revised the manuscript.

\section{CONFLICT OF INTEREST}

There are no conflicts of interest in this study.

\section{FUNDING AND SPONSORSHIP}

None

\section{ACKNOWLEDGEMENT}

This study was conducted as part of the requirements for MD degree from the Goldman Medical School at the Faculty of Health Sciences, Ben-Gurion University of the Negev, Beer-Sheva, Israel.

\section{REFERENCE}

Abokaf H, Shoham-Vardi I, Sergienko R, Landau D, Sheiner E (2018). In utero exposure to gestational diabetes mellitus and long term endocrine morbidity of the offspring, Diabetes Res Clin. 231-235. https://doi.org/10.1016/j.diabres.2018.09.003.

Barker DJP, Eriksson JG, Forsen T, Osmond $C$ (2002). Fetal origins of adult disease: Strength of effects and biological basis, Int $\mathrm{J}$ Epidemiol, 31(6): 1235-1239. https://doi.org/10.1093/ije/31.6.1235.

Boney CM, Verma A, Tucker R, Vohr BR (2005). Metabolic syndrome in child- hood: Association with birth weight, maternal obesity, and gestational diabetes mellitus, Pediatrics, 115(3). https://doi.org/10.1542/peds.2004-1808.

De Boo HA, Harding JE (2006). The developmental origins of adult disease (Barker) hypothesis, Aust N Z J Obstet Gynaecol, 46(1): 4-14. https://doi.org/10.1111/j.1479-828X.2006.00506.x.

Bureau C, Statistics OF (2019). 31.12.2018 By District, Sub-District and Population Religion, (3): 3-6.

Cecatti JG, Correa-Silva EPB, Milanez H, Morais S, Souza JP (2008). The associations between inter-pregnancy interval and maternal and neonatal outcomes in Brazil. Matern Child Health J. 12(2): 275-281. https://doi.org/10.1007/s10995-007-0219-y.

Class QA, Rickert ME, Oberg AS, Sujan AC, Almqvist C,Larsson $\mathrm{H}$, Lichtenstein $\mathrm{P}$, et al. (2017). Within-family analysis of interpregnancy interval and adverse birth outcomes, Obstetrics and Gynecology, 130(6): 1304-1311. https://doi.org/10.1097/AOG.0000000000002358.

Conde-Agudelo A, Rosas-Bermúdez A, Kafury-Goeta AC (2006). Birth spacing and risk of adverse perinatal outcomes: A meta-analysis. J Am Med Assoc. 1809-1823. https://doi.org/10.1001/jama.295.15.1809.

Eshkoli T, Wainstock T, Sheiner E, Beharier O, Fraenkel M, Walfisch A (2019). Maternal hypothyroidism during pregnancy and the risk of pediatric endocrine morbidity in the offspring, Am J Perinatol, 36(9): 975-980. https://doi.org/10.1055/s-0038-1675834 .

Kaul P, Bowker SL, Savu A, Yeung OR, Donovan LE, Ryan EA (2019). Asso- 
Seidman et al./ Inter-pregnancy interval on long-term endo-metabolic health

ciation between maternal diabetes, being large for gestational age and breast-feeding on being overweight or obese in childhood, Diabetologia, 62(2): 249-258. https://doi.org/10.1007/s00125-018-4758-0.

Li S, Hua J, Hong $\mathrm{H}$, Wang Y, Zhang J (2018). Interpregnancy interval, maternal age, and offspring's BMI and blood pressure at 7 years of age, $\mathrm{J}$ Hum Hypertens, 32(5): 349-358. doi: 10.1038/s41371-018-0035-4.

van Lieshout N, Oostvogels AJJM, Gademan MGJ, Vrijkotte TGM (2017). Maternal early pregnancy lipid profile and offspring's lipids and glycaemic control at age 5-6 years: The ABCD study, Clin Nutr, 36(6): 1628-1634. https://doi.org/10.1016/j.clnu.2016.10.010 .

Mahande MJ, Obure J (2016). Effect of interpregnancy interval on adverse pregnancy outcomes in northern Tanzania: A registry-based retrospective cohort study, BMC Pregnancy and Childbirth, 16(1): 1-9. https://doi.org/10.1186/s12884-016-0929-5.

Mignini LE, Carroli G, Betran AP, Fescina R, Cuesta C, Campodonico L, De Mucio B, et al. (2016). Interpregnancy interval and perinatal outcomes across Latin America from 1990 to 2009: A large multi-country study, Bjog-Int J Obstet Gy, 123(5): 730737. https://doi.org/ 10.1111/14710528.13625.

Oostvogels AJJM, Stronks K, Roseboom TJ, van der post JAM, van Eijsden M, Vrijkotte TGM (2014). Maternal prepregnancy BMI, offspring's early postnatal growth, and metabolic profile at age 5-6 years: The ABCD study, J Clin Endocr Metab, 99(10): 3845-3854. https://doi.org/10.1210/jc.2014-1561.
Paz Levy D, Sheiner E, Wainstock T, Sergienko R, Landau D, Walfisch A (2017). Evidence that children born at early term (37-38 6/7 weeks) are at increased risk for diabetes and obesityrelated disorders, Am J Obstet Gynecol: 588.e1-588.e11. https://doi.org/10.1016/j.ajog.2017.07.015.

Ratzon R, Sheiner E, Shoham-Vardi I (2011). The role of prenatal care in recurrent preterm birth, Eur J Obstet Gynecol Reprod Biol. Elsevier Ireland Ltd, 154(1): 40-44. https://doi.org/10.1016/j.ejogrb.2010.08.011.

Regan AK, Ball SJ, Warren JL, Malacova E, Marston C, Nassar N, Leonard H, et al. (2019). Reply to "Sibling comparison design in birth-spacing studies". Am J Epidemiol, 188(1): 22-23. https://doi.org/10.1093/aje/kwy187.

ShahA, Faundes A, Machoki M, Bataglia V, Amokrane F, Donner A, Mugerwa K, et al. (2008). Methodological considerations in implementing the WHO Global Survey for Monitoring Maternal and Perinatal Health, B World Health Organ, 86(2): 126-131. https://doi.org/10.2471/BLT.06.039842.

Spiegel E, Shoham-Vardi I, Sergienko R, Landau D, Sheiner E(2019). The association between birth weight at term and long-term endocrine morbidity of the offspring, J Matern-Fetal Neo M. Informa UK Ltd. 32(16): 2657-2661. https://doi.org/10.1080/14767058.2018.1443440.

Tran BX, Dang KA, Le HT, Nguyen LH, Tran TH, Latkin CA, Ho CSH, et al. (2019). Global evolution of obesity research in children and youths: Setting priorities for interventions and policies, Obes Facts, 12(2): 137-149. https://doi.org/10.1159/oo0497121. 